\title{
Surface Engineering by Plasma Techniques of DLC for Medical Materials and B lood-compatibility Evaluation
}

\author{
Tatsuyuki Nakatani , Keishi Okamoto, Yuki Nitta, Akira Mochizuki", \\ Hideo Hoshi $^{* *}$ and Akihiko Homma ${ }^{* *}$ \\ Toyo Advanced Technologies Co., Ltd. \\ 5-3-38 Ujina-higashi Minami-ku Hiroshima 734-8501, Japan \\ "Department, of Bio-Medical engineering, Tokai University, \\ 317 Nishino Numazu Shizuoka 410-0395, Japan \\ ${ }^{* *}$ National Cardiovascular Center \\ 5-7-1 Fujishiro-dai, Suita, Osaka 565-8565, Japan
}

\begin{abstract}
The metallic medical devices has high shape stability and durability, but the effects of chemical changes in surface by ingredients inside the body are not negligible. Therefore, metallic medical devices are given surface treatment with carbonaceous thin films or the like. Accordingly, with the purpose of improving the biocompatibility of Diamond-like carbon (DLC) films, we experimented with fabricating biomimetic multifunctional DLC films whose zeta potential is controlled. As a result, we discovered that reducing the $\mathrm{Si}$ content is effective for introduction of functional groups onto DLC films. We also succeeded in controlling the zeta potential on DLC surface, by controlling the amounts of anionic and cationic groups introduced. In addition, in assessing the blood compatibility, we found that the DLC surface's zeta potential correlates with the platelet adhesion characteristic and the blood coagulation characteristic; we thus discovered a possibility for optimizing DLC surface engineering. This indicated the possibility that biomimetic materials, multifunctional DLC films using plasma surface treatment techniques could be applied to develop stents and artificial organs with higher biocompatibility.
\end{abstract}

Keywords: Diamond-Like-Carbon, silicon, plasma treatment, medical devices

\section{Introduction}

Amorphous carbon coatings have various advantageous characteristics, such as being deposited at low temperature using plasma enhanced chemical vapor deposition (PECVD), being mechanically and chemically stable, and being superior frictional characteristics. Their industrial application has therefore been advancing at a rapid rate $[1,2]$. For the future, the emphasis of carbon coating application looks set to shift toward finer materials such as electronic materials and biomaterials. DLC films form extremely smooth, flat, inert surfaces, and much is expected of them as a surface treatment for medical-application materials, where interaction with biological matter is to be avoided [3-9]. For these reasons, greater control over structure and surface functionalization is likely to become important in thin film fabrication.

We have previously reported on the application of DLC films to drug-eluting stents for coronary arteries $[10,11]$. As Fig. 1 shows, coronary artery stents take the form of a metallic mesh tube. They are medical devices that are used for treatment of symptoms arising from stenosis or occlusion of coronary arteries, and secure the flow of blood by being left in place after insertion to the disease part. Our DLC films for stents was developed as a highly-tenacity, $\mathrm{Si}$ concentration gradient-type DLC films that is able to track deformation of the 


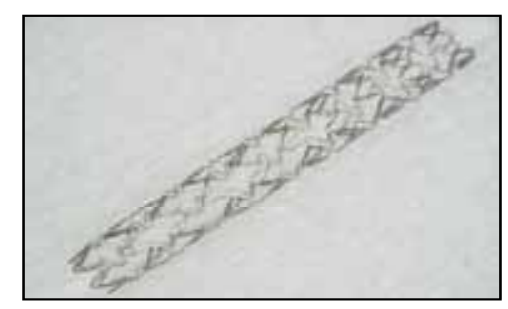

Fig. 1 Photograph of coronary artery stent (diameter $\approx 3.0 \mathrm{~mm}$, length $\approx 15 \mathrm{~mm}$ )

base material and does not have cracks [10]. Further, the surface modification method that we developed employs introduction of functional groups by plasma irradiation onto the DLC films. Specifically this is a method whereby firstly, reactive sites of carbon radicals or the like are formed on the DLC films through plasma irradiation, then the surface is exposed to the atmosphere so as to generate functional groups such as hydroxyl groups, which are then used as footholds for coating with drug-containing biodegradable polymer.

However, as the drug-containing biodegradable polymer is designed to elute into the blood for several weeks after being left inside a artery, the DLC surface will ultimately come into contact with endothelial cells or blood. Also, non-coated stents, which do not use drugs or biomolecules, have been increasingly under review in recent years. Therefore, imparting biocompatibility on DLC films ranks as an important task in order to functionalize the surfaces of stents and artificial organs.

Accordingly, we have studied the possibility of imparting biocompatibility by controlling the functional groups that are generated on the surface of DLC films using plasma surface treatment techniques. We believe that thereby the creation of a new DLC films that is both durable and functional can be expected. We are focusing particularly on interaction between the DLC surface and biomatter. Cells are held to generally be negatively charged, and the zeta potential of their surfaces varies with each cell. Hence it is surmised that it will be possible to control interaction with DLC surface and cells that wishes to cause to adhere by having same zeta potential or be not adsorbed. Therefore, controlling zeta potential is an important requirement for achieving a DLC surface that is inert with regard to cells. The zeta potential control method that we focused on was introduction of anionic groups and cationic groups by using plasma surface treatment. Fig. 2 is a schematic of a DLC surface with functional groups introduced. Anions are negatively charged ions, and cations are positively charged ions. Hence, if the ratio of composition of these functional groups that are introduced can be controlled at the DLC surface, it should be possible to control the zeta potential and thus to have particular cells selectively adhere or be not adsorbed by the DLC surface.

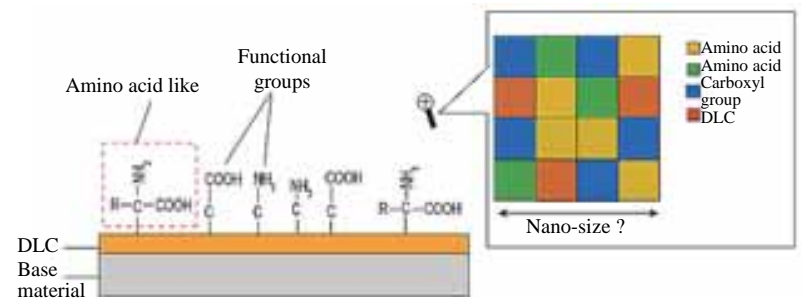

Fig. 2 Schematic of DLC surface with functional groups introduced

In this study, we attempted plasma surface treatment on DLC films so as to experiment with introduction of functional groups to its surface. We also measured the zeta potential of DLC film with anionic groups and cationic groups introduced. Further, by introducing anionic groups and cationic groups simultaneously on a DLC surface, we created a biomimetic DLC films with zwitterions structure. And then, we investigated the relation between zeta potential and blood compatibility by various conditions on the DLC films.

\section{Experimental method}

\subsection{Preparation of DLC thin film}

To investigate, $10 \mathrm{~mm}$ square, $1 \mathrm{~mm}$ thick pieces of cobalt chrome (CoCr) alloy were used as the samples. An ionized deposition apparatus was used for deposition the DLC films. Plasma was generated by introducing argon (Ar) and benzene $\left(\mathrm{C}_{6} \mathrm{H}_{6}\right)$ gases serving as the ion sources into a plasma generation source that used thermions obtained from a filament installed inside a process chamber.

First of all, the samples was placed inside the chamber of the ionized deposition apparatus, and $\mathrm{Ar}$ gas was introduced in so as to render the pressure $10^{-1}$ to $10^{-3} \mathrm{~Pa}$. Then bombardment cleaning was implemented for about 30 minutes by discharge. 
Following that, tetramethylsilane $\left(\mathrm{Si}\left(\mathrm{CH}_{3}\right)_{4}\right)$ and $\mathrm{C}_{6} \mathrm{H}_{6}$ were passed in, adjusting the deposition pressure $10^{-1} \mathrm{~Pa}$. While introducing gas in continuously at a rate of $30 \mathrm{ml} / \mathrm{min}, \mathrm{C}_{6} \mathrm{H}_{6}$ gas was ionized and a DLC films was deposited. An amorphous DLC film, with a thickness $30 \mathrm{~nm}$ and mainly consisting of silicon (Si), carbon (C) and hydrogen $(\mathrm{H})$, was deposited on the surface of the sample. DC bias voltage was $-1.5 \mathrm{kV}$. Also, the temperature of the substrate during the thin film deposition was about $160^{\circ} \mathrm{C}$.

\subsection{Plasma surface treatment}

There follows a description of the plasma surface treatment apparatus. The chamber interior was evacuated to below $5 \mathrm{~Pa}$ with a rotary pump, the pressure being measured with a Pirani gauge. The process chamber was connected to an RF power source with maximum output $300 \mathrm{~W}$ (Adtec Plasma Technology, model AX-300; frequency 13.56 MHz), and RF electric power of $30 \mathrm{~W}$ was injected as the plasma generating power. Capacitively-coupled plasmas was generated by means of two parallel plate electrodes. The operation gases were oxygen, Ar and ammonia $\left(\mathrm{NH}_{3}\right)$, which were introduced into the chamber by a mass flow controller. The duration of plasma irradiation was 15 seconds, or 30 seconds for continuous treatment with the gas varied.

\subsection{Surface analysis}

The functional groups introduced on the surface of the DLC films were analyzed by using X-ray photoelectron spectroscopy (XPS). The instrument used for this was a JEOL JPS9010 XPS apparatus. Non-monochromatized AlKa rays $(1286.3 \mathrm{eV})$ were used as the $\mathrm{X}$-ray source, with the accelerating voltage set at $12.5 \mathrm{kV}$ and the emission current at $15 \mathrm{~mA}$. The measurements were made at room temperature, under pressure of $8.0 \times 10^{-7} \mathrm{~Pa}$. The main peaks of the C1s spectra obtained were corrected by adjustment to the binding energy of amorphous carbon, $284.3 \mathrm{eV}$ [12], and the backgrounds were eliminated using the Shirley method [13]. The measurements focused on the carboxyl groups. To measure the carboxyl groups, peak splitting was carried out by taking peaks on the $4.3 \mathrm{eV}$ high coupling energy side of the $\mathrm{C} 1 \mathrm{~s}$ peak as being $\mathrm{O}=\mathrm{C}-\mathrm{O}$ bonds. Further, the C1s peaks were split by assuming
$287.1 \mathrm{eV}$ for the $\mathrm{C}=\mathrm{O}$ bonds and $285.8 \mathrm{eV}$ for the C-O bonds [14]. To cross-check that the surface of the DLC film had been modified with functional groups, we measured the surface's zeta potential and assessed the correlation with the XPS analysis results. An Otsuka Electronics ELS-Z was used to measure the zeta potential. The measurement method was as follows. Plasma-irradiated DLC films was attached to a chamber for plate sample, and the chamber interior was filled with monitoring particles dispersed in purified water. Electrophoresis of the monitoring particles was implemented for each depthwise level of the chamber, and the apparent velocity distribution in the chamber interior was determined. The conditions for implementation of electrophoresis were $17.33 \mathrm{~V} / \mathrm{cm}$ average field, $1.02 \mathrm{~mA}$ average current. The zeta potential of the DLC films surface was derived by using the Mori-Okamoto equation [15] to analyze the apparent velocity distribution obtained.

\subsection{Evaluation of blood compatibility}

To assess blood compatibility, we assessed two items: platelet adhesion characteristic and blood coagulation characteristic. Fig. 3 is a schematic diagram of the process of assessing the thrombin-antithrombin III complexes (TAT) production quantity. To determine the blood coagulation characteristics, heparinized whole blood $(1,000 \mu \mathrm{l})$ was pipetted into a chamber with $2,613 \mathrm{~mm}^{2}$ of surface area (40-mm diameter) and the chamber was rotated vertically at $47 \mathrm{rpm}$ at 37 ${ }^{\circ} \mathrm{C}$ for 120 minutes to avoid sedimentation of blood cells during incubation. After incubation, the blood was moved to a micro-test-tube, followed by addition of $10 \%$ sodium citrate solution. The blood was centrifuged at 3,000 rpm for $10 \mathrm{~min}$ to obtain

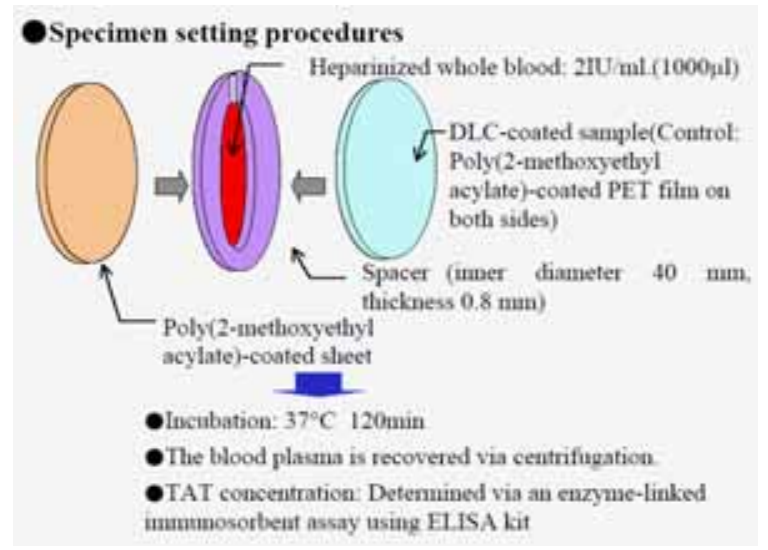

Fig. 3 Schematic of TAT production quantity assessment 
plasma. The level of TAT in the blood plasma was evaluated using an ELISA kit (TAT-EIA, Enzyme Research Laboratories) and microplate reader (Thermo Max, Molecular Device Corporation). The amount of platelets adhering to the samples was measured in the following manner. $130 \mu \mathrm{l}$ of blood plasma $\left(1 \times 10^{5}\right.$ platelets $\left./ \mu \mathrm{l}\right)$ was placed on the poly(2-methoxyethyl acylate)-coated PET film and incubated for 60 minutes at $37{ }^{\circ} \mathrm{C}$. After the film was washed, it was immersed in $1 \%$ glutaraldehyde in phosphate-buffered saline (PBS) for 60 minutes at $4{ }^{\circ} \mathrm{C}$ to fix the adherent platelets. The sample was freeze-dried and sputter-coated using platinum, prior to observation by scanning electron microscopy (JSM-840, JEOL).

\section{Results and discussion}

3.1. Control of introduction of functional groups to DLC surface

We studied efficient introduction of functional groups to the DLC surface. For the measurements, we used an angle-resolved XPS analysis method [16]. Using this method, we first analyzed the composition of Si-doped DLC, then used $\mathrm{Ar}$ and $\mathrm{O}_{2}$ plasmas to form functional groups on the DLC surface and assessed the correlation with the amount of doped Si. First, the $\mathrm{O}=\mathrm{C}-\mathrm{O}$ bonds were determined from C1s spectrum for DLC coated sample having different Si contents. Also, SiC and Si-O were determined from Si2p spectrum.

From the results, the $\mathrm{O}=\mathrm{C}-\mathrm{O}$ bonds were observed to decrease as the $\mathrm{Si}$ contents increased. The ratio of intensity of the $\mathrm{O}=\mathrm{C}-\mathrm{O}$ bonds relative to the carbon at such times were: $\mathrm{Si}(0 \%)=5.4 \%$, Si $(3 \%)=5.0 \%$, Si $(19 \%)=4.0 \%$, and Si $(28.5 \%)$ $=1.9 \%$ (Fig. 4).

Also, comparison of $\mathrm{SiO}_{2} / \mathrm{Si}$ in the DLC having different Si contents gave the composition ratio $\mathrm{Si}$ $(3 \%)=0.64$, Si $(19 \%)=0.55$, and Si $(28.5 \%)=$ 0.48 . Thus, it was found that the $\mathrm{SiO}_{2} / \mathrm{Si}$ composition ratio did not vary greatly with the Si content. Furthermore, the surfaces were measured and assessed with greater sensitivity by using angle-resolved XPS analysis and varying the incidence angle, specifically by making the X-rays incident at $0^{\circ}$, which is perpendicular to the sample surface, and at the slanted angles $37.5^{\circ}$ and $75^{\circ}$ (Fig. 5). It can be seen from that figure that as the incidence angle increases, the C1s spectrum decrease and the $\mathrm{Si}$ oxides increase. This is probably because $\mathrm{O}_{2}$ radicals contributed greatly to oxidation of the Si. It could also be explained that oxidation of the $\mathrm{Si}$ were easier than carbon, because the electro negativity of $\mathrm{Si}$ is 1.9 whereas that of carbon is 2.55 .

From the above results, it was evident that in surface treatment using oxygen plasma, the introduction of functional groups via oxidation of carbon in the DLC surface was influenced by the concentration of Si in the DLC surface.

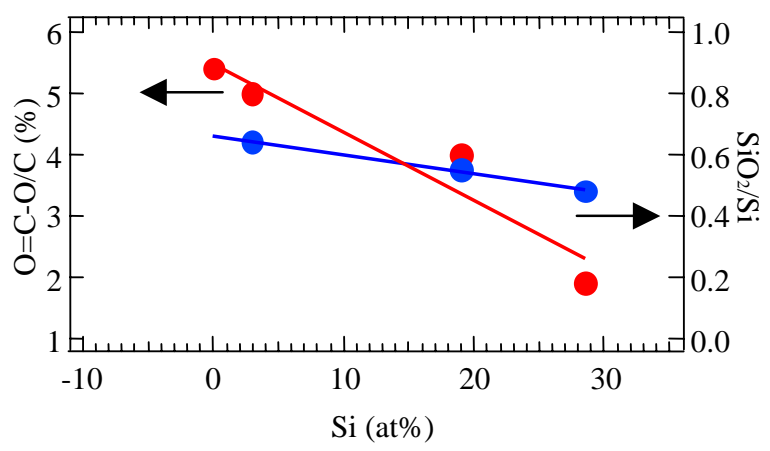

Fig. 4 XPS analysis of $\mathrm{C} 1 \mathrm{~s}$ and $\mathrm{Si} 2_{\mathrm{P}}$ spectra of Si-DLC with differing Si concentrations after plasma irradiation

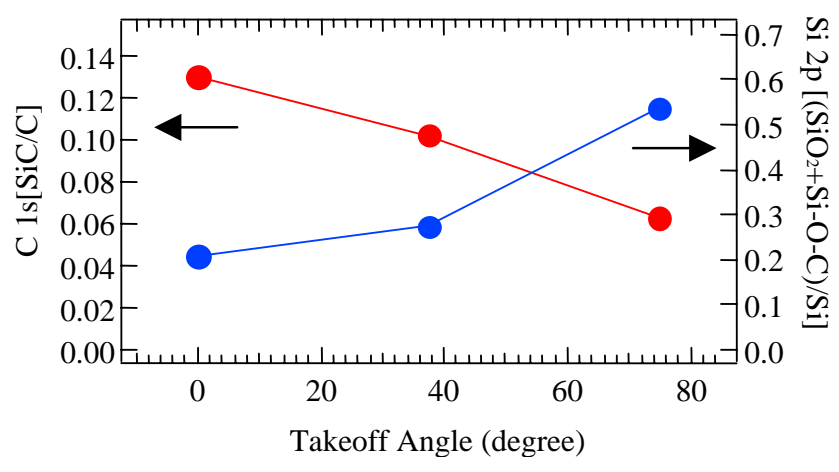

Fig. 5 XPS analysis of $\mathrm{C} 1 \mathrm{~s}$ and $\mathrm{Si} 2_{\mathrm{P}}$ spectra of $28.5 \%$-Si-DLC using $\mathrm{X}$-ray incidence angle-resolved

\subsection{Zeta potential control of DLC surface}

Fig. 6 shows the C1s waveforms were observed when the sample was treated by $\mathrm{O}_{2}$ plasma. The $\mathrm{C} 1 \mathrm{~s}$ peaks have been separated into four: $\mathrm{C}-\mathrm{C}, \mathrm{C}-\mathrm{O}$, $\mathrm{C}=\mathrm{O}$ and $\mathrm{O}-\mathrm{C}=\mathrm{O}$. Table 1 shows the ratios of $\mathrm{O}=\mathrm{C}-\mathrm{O}$ and $\mathrm{N}$ in $\mathrm{C} 1 \mathrm{~s}$ that were obtains as a results of implementing the plasma surface treatment. These results show that by selecting the gas type it was possible to control the ratio of $\mathrm{N}$ to $\mathrm{C} 1 \mathrm{~s}$ in a range from 0.004 up to 0.052 , and to control the ratio of $\mathrm{O}=\mathrm{C}-\mathrm{O}$ to $\mathrm{C} 1 \mathrm{~s}$ in a range from 0.010 up to 0.052 . From these facts it is evident that the $\mathrm{C}-\mathrm{C}$ bonds or C-H bonds of the DLC surface are braked 
by radicals, electrons or ions in the plasma, so that oxidizing reactions such as $\mathrm{C}-\mathrm{O}, \mathrm{C}=\mathrm{O}, \mathrm{O}=\mathrm{C}-\mathrm{O}$, or nitriding reactions, are promoted.

Furthermore, when $\mathrm{NH}_{3}$ plasma treatment was implemented, peaks originating from nitrogen were found in the XPS measurements. These nitrogen peaks appeared in the neighborhood of $400 \mathrm{eV}$, which coincides with amino group peaks, which are present at $400 \pm 1 \mathrm{eV}$. It is therefore probable that amino groups were generated on the surface of the DLC films.

Next, we assessed the correlation with the zeta potential. Fig. 7 shows the correlative relationship between the $\mathrm{N} / \mathrm{O}=\mathrm{C}-\mathrm{O}$ ratio and the zeta potential. This verified that the larger the $\mathrm{N} / \mathrm{O}=\mathrm{C}-\mathrm{O}$ ratio, the greater the zeta potential was, and hence that there was a correlation with the amounts obtained by XPS for the two functional group types. In other words, it was found that the DLC surface was modified by both anionic and cationic functional groups. This would indicate that an zwitterion structure was obtained in the DLC surface. It was also found that the zeta potential was negative with an untreated DLC film, but became positive when the $\mathrm{N} / \mathrm{O}=\mathrm{C}-\mathrm{O}$ ratio became large. Where carboxyl groups $(-\mathrm{COOH})$ were present, they generally split as follows:

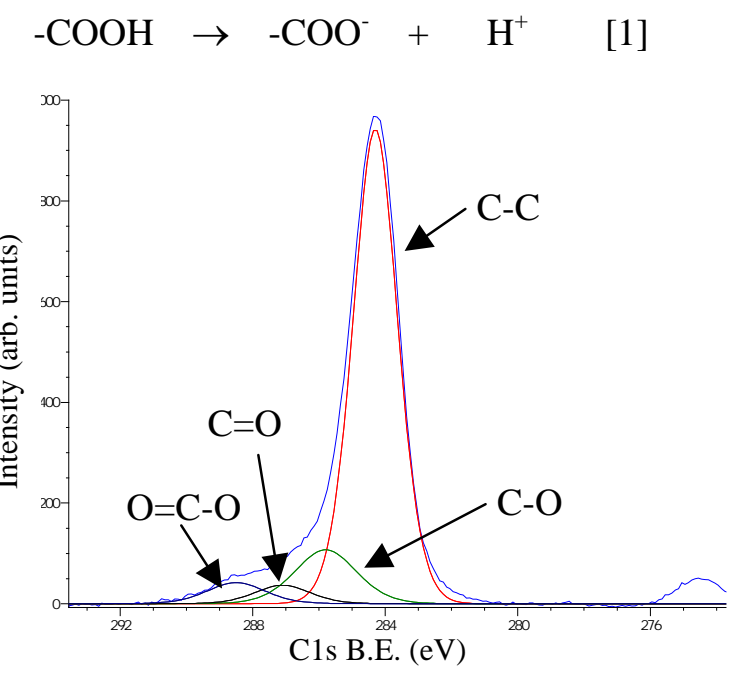

Fig. 6 XPS spectra of DLC surface in C1s binding energy region after $\mathrm{O}_{2}$ plasma irradiation

Table 1 Plasma treatment gases, and functional group ratio obtained therewith

\begin{tabular}{lcccccc}
\hline First gas & $\mathrm{O}_{2}$ & $\mathrm{Ar}$ & $\mathrm{NH}_{3}$ & $\mathrm{Ar}$ & $\mathrm{O}_{2}$ & $\mathrm{DLC}$ \\
Second Gas & - & - & - & $\mathrm{NH}_{3}$ & $\mathrm{NH}_{3}$ & - \\
\hline$[\mathrm{N}] /[\mathrm{C}]$ & 0.004 & 0.006 & 0.126 & 0.043 & 0.146 & 0.004 \\
{$[\mathrm{COO}] /[\mathrm{C}]$} & 0.048 & 0.052 & 0.010 & 0.033 & 0.010 & 0.017 \\
{$[\mathrm{~N}] /[\mathrm{COO}]$} & 0.08 & 0.11 & 12.48 & 1.31 & 15.01 & 0.26 \\
\hline
\end{tabular}

and the sample became negatively charged. This was probably the cause of the zeta potential declining as the - $\mathrm{COOH}$ increased. Also, the fact that the zeta potential became positive was probably due to the influence of amino groups $\left(-\mathrm{NH}_{3}{ }^{+}\right)$in which protons had bonded. The present experiments did not examine the influence of hydroxyl groups and carbonyl groups on the zeta potential; this will need to be studied in the future. Such study will need to include elucidation of domain formation.

The foregoing results demonstrate that the surface potential can be controlled by the ratio of composition of carboxyl and amino groups that are introduced, which indicates a possibility of imparting tissue cell compatibility to the DLC films.

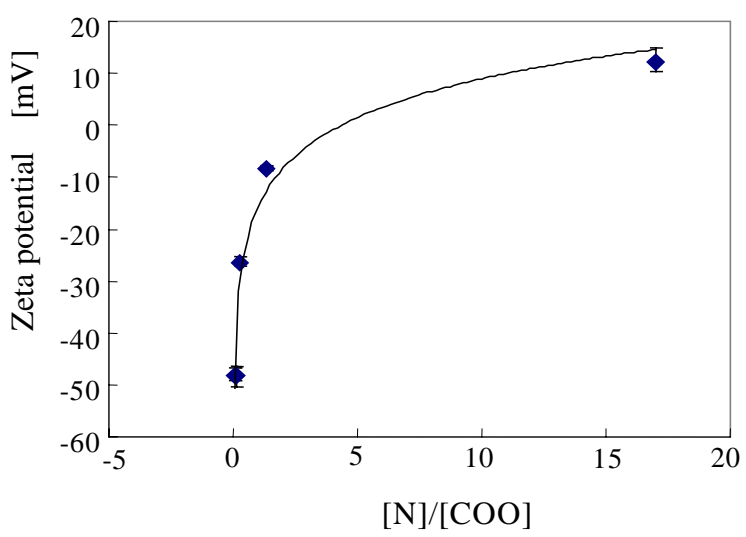

Fig. $7[\mathrm{~N}] /[\mathrm{COO}]$ ratio versus zeta potential

\subsection{Fabrication of biomimetic DLC and evaluation of blood compatibility}

Fig. 8 shows the relation between the ratio of the $\mathrm{N}$ component to the $\mathrm{O}=\mathrm{C}-\mathrm{O}$ component on the one hand, and compatibility with regard to the amounts of TAT produced and amounts of platelets adhering, on the other. The $\mathrm{N}$ component to $\mathrm{O}=\mathrm{C}-\mathrm{O}$ component ratio $\mathrm{N} / \mathrm{O}=\mathrm{C}-\mathrm{O}$ in the figure was derived using $(\mathrm{N} / \mathrm{C}) /(\mathrm{O}=\mathrm{C}-\mathrm{O} / \mathrm{C})$. As this figure shows, the greater the $\mathrm{N} / \mathrm{O}=\mathrm{C}-\mathrm{O}$ ratio, the higher the compatibility with regard to the blood coagulating factors. Thus, it was found that optimal compositional ratio also exist in an zwitterion structure, resulting in the film showing superior blood compatibility if the zeta potential is high. However, it is not clear why the film exhibits superior compatibility with regard to both platelets adhesion and blood coagulating factors when the $\mathrm{O}=\mathrm{C}-\mathrm{O} / \mathrm{C}$ component ratio is low and the $\mathrm{N} / \mathrm{C}$ is 
high. But $\mathrm{O}=\mathrm{C}-\mathrm{O} / \mathrm{C}$ is a functional group possessing a negative charge, while $\mathrm{N} / \mathrm{C}$ is a functional group possessing a positive charge. Thus, it might be that influence to blood-compatibility is exerted by the amounts of the positive and negative charges, and by the distances between the functional groups, on the surface of the carbonaceous films.

The foregoing results indicate that by optimizing the amounts of carboxyl and amino groups introduced to the DLC surface we have succeeded in developing an zwitterion structure. This biomimetic DLC film may be suggested as a new biocompatible material with high blood compatibility compared to conventional DLC.

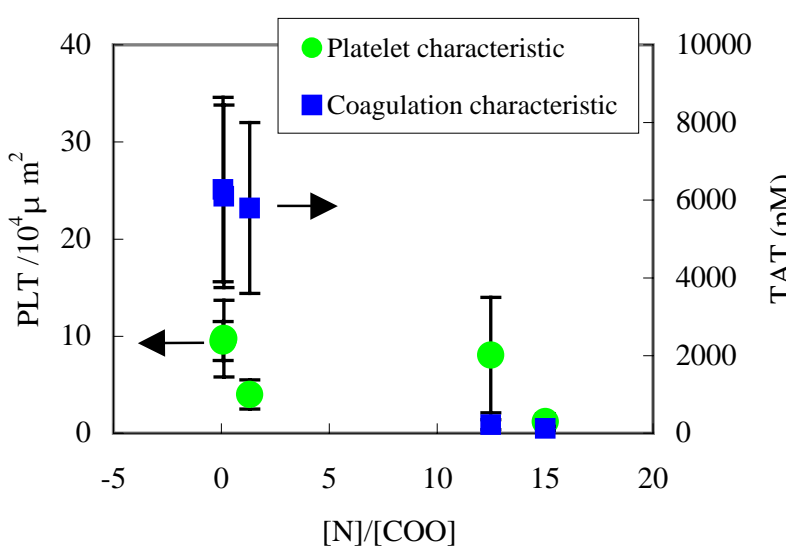

Fig. 8 Relation between [N]/[COO] ratio and blood coagulation and platelet adhesion characteristics

\section{Conclusion}

We established that reducing the $\mathrm{Si}$ content is effective for introduction of carboxyl and other functional groups to DLC surface. We also found that the amounts of anionic and cationic groups introduced can be controlled by altering the plasma irradiation conditions. Further, we succeeded in obtaining zeta potentials spread over a wide range by varying the amounts of functional groups introduced to the DLC surface. In addition, we found that by optimizing the amounts of carboxyl and amino groups introduced to the DLC surface, its blood compatibility is enhanced compared to untreated DLC. Through the experiments, we discovered that there is a possibility that multifunctional DLC film using plasma surface treatment technique can be used on the surfaces of coronary artery stents, and other equipment.

\section{Acknowledgements}

We wish to thank Dr. Shuzou Yamashita of Japan Stent Technology, the promoter of the joint project on stents, for his multifaceted assistance to us in carrying out our research. We also acknowledge gratefully the kind advice we received on plasma techniques from Emeritus Professor Noriyoshi Sato of Tohoku University, on film surface evaluation methods from Professor Takayuki Takahagi of Hiroshima University.

\section{References}

1. T. Nakatani, K. Okamoto, A. Araki, T. Washimi, New Diamond and Frontier Carbon Technology, 16 (2006) 187.

2. C. Weissmantel, K. Bewilogua, K. Breuer, D. Dietrich, U. Ebersbach, H. J. Erler, B. Rau and G. Reisse, Thin Solid Films, 96 (1996) 31.

3. D. P. Maguire, Diamond and Related materials, 14 (2005) 1277.

4. B. Balram, et al., Catheterization and Cardiovascular Interventions, 67 (2006) 698.

5. T. Hasebe, A. Kamijo, A. Hotta, K. Takahashi, T. Suzuki, Chemistry and Chemical Industry, 59 (2006) 1064.

6. R. Hauert, Tribology International, 37 (2004) 991.

7. R. Hauert, Diam. Relat. Mater., 12 (2003) 583.

8. M. I. Jones, I. R. McColl, D. M. Grant, K. G. Parker, T. L. Parkar, J. Biomed. Mater. Res., 52 (2000) 413.

9. D. Antoniucci, et al., Am. J. Cardiol., 85 (2000) 821.

10. T. Nakatani, K. Okamoto, I. Omura, S. Yamashita, J. Photopolym. Sci. Technol., 20 (2007) 221.

11. K. Okamoto, T. Nakatani, S. Yamashita, S. Takabayashi, T. Takahagi , Surface and Coating Technology, in press..

12. Handbook of X-ray Photoelectron Spectroscopy , JEOL, (1991) p.155.

13. A. Jablonsk, Surf. Interface Anal., 23 (1995) 13.

14. J. S. Brinen,, S. Greenhouse, L. Pinatti, Surf.Interface Anal., 17 (1991) 63.

15. H. Mori, H. Okamoto, Fusen, 27 (1980) 117.(in Japanese)

16. S. Takabatashi, K. Motomitsu, T. Takahagi, A. Terayama, K. Okamoto and T, Nakatani, J. Appl. phys., 101 (2007) 103542. 